\title{
Diversidad alfa, beta y co-ocurrencia de especies de flebotomineos (Diptera: Psychodidae) en Calakmul, Campeche, México
}

\author{
Alpha, beta diversity and co-occurrence of phlebotomine sand flies (Diptera: Psychodidae) from \\ Calakmul, Campeche, Mexico
}

Eduardo A. Rebollar-Téllez ${ }^{1,2, *}$ y David A. Moo-Llanes ${ }^{3}$

\begin{abstract}
${ }^{1}$ Universidad Autónoma de Nuevo León (UANL), Facultad de Ciencias Biológicas, Departamento de Zoología de Invertebrados, Laboratorio de Entomología Médica. San Nicolás de los Garza, Nuevo León, México. E-mail correspondencia: eddiethelittle@gmail.com* ORCID: http://orcid.org/0000-0002-8173-3550

${ }^{2}$ Centro de Investigación en Ciencias de la Salud (CIDICS), Universidad Autónoma de Nuevo León, San Nicolás de los Garza, Nuevo León, México.

${ }^{3}$ Centro Regional de Investigación en Salud Pública, Instituto Nacional de Salud Pública, Tapachula, Chiapas, México. E-mail:davidmooll@gmail.com ORCID: http://orcid.org/0000-0001-5864-4990
\end{abstract}

\section{ZooBank: urn:lsid:zoobank.org:pub: 211C28BE-5886-47D4-9171-9DA66A78DB0B https: / / doi.org/10.35249/ rche.46.2.20.13}

Resumen. Las leishmaniasis son un conjunto de manifestaciones clínicas de gran importancia en México. El objetivo del presente estudio es analizar la diversidad y co-ocurrencia de flebotomineos en Calakmul, Campeche. Se capturaron flebotomineos en siete localidades durante el periodo de 20012007, con diferentes tipos de trampas. En total se recolectaron 17.405 flebotomineos representados por nueve géneros y 17 especies. La mayor diversidad alfa se presentó en Dos Lagunas Sur y La Guadalupe, mientras que la mayor dominancia se dio en Unión 20 de Junio y Arroyo Negro. La mayor equidad se presentó en Dos Naciones y La Virgencita. Se encontró que la mayor diversidad beta se presentó entre en Unión 20 de Junio-La Guadalupe, La Guadalupe-La Virgencita y Dos Naciones-Dos Lagunas Sur con un 99\%. Finalmente, el análisis de co-ocurrencia de especies, no permitió inferir alguna relación significativa entre flebotomineos. En conclusión, se reportan dos nuevos registros para Campeche: Micropygomyia (Micropygomyia) cayennensis maciasi y Dampfomyia (Coromyia) steatopyga. Los estimadores de biodiversidad son efectivos para explicar la riqueza y estructura de la comunidad, se argumenta la utilidad del estadístico $Q$, que, al no ser influenciado por especies abundantes o especies raras, resultaría en una buena estimación de la heterogeneidad.

Palabras clave: Campeche, diversidad, leishmaniasis, Phlebotominae, riqueza.

Abstract. Leishmaniases are a group of clinical manifestations that represent a health problem in Mexico. We analyzed the diversity of phlebotominae sand fly in seven localities of the municipality of Calakmul, Campeche. We collected sandflies during 2001-2007 with three methods: light CDC traps, mice-baited Disney traps, and human-baited Shannon traps. A total of 17405 phlebotomine sand flies were collected, representing nine genera and 17 species. Communities with the highest species richness were Once de Mayo and Dos Naciones. Alpha diversity was found to be highest in Dos Lagunas Sur and La Guadalupe, whereas the highest dominance was found in Unión 20 de Junio and Arroyo Negro. Likewise, the highest record for species eveness was detected in Dos Naciones and La Virgencita. In general, it was found that

Recibido 1 Abril 2020 / Aceptado 6 Mayo 2020 / Publicado online 19 Mayo 2020

Editor Responsable: José Mondaca E. 
beta diversity exhibited the highest values (share species) $99 \%(\approx 17$ species) between Union 20 de Junio-La Guadalupe, as well as between La Guadalupe-La Virgencita and Dos Naciones-Dos Lagunas Sur, respectively. Using C-score analysis, it was found that co-occurrence of species at that spatial scale was found to be random, with not significant scores for either an aggregated or dispersed occurrence. In conclusion, two are new records for the state of Campeche: Micropygomyia (Micropygomyia) cayennensis maciasi and Dampfomyia (Coromyia) steatopyga. Biodiversity estimators are effective in explaining the richness and structure of the community, arguing the utility of the $\mathrm{Q}$ statistic, which, not being influenced by abundant species or rare species, would result in a good estimate of heterogeneity.

Key words: Campeche, diversity, leishmaniasis, Phlebotominae, richness.

\section{Introducción}

Las leishmaniasis representan una amplia variedad de manifestaciones clínicas, entre las que destacan la forma cutánea, muco-cutánea y la visceral, aunque también existen cuadros clínicos inusuales e intermedios. Los casos de leishmaniasis se han registrado hasta ahora en 98 países (Alvar et al. 2012) y se estima que hay de 1,5 a 2 millones de personas infectadas a nivel mundial y unos 400.000 casos nuevos con una población en riesgo de 350 millones de personas (Ashford 1992). Aunque las cifras anteriores sobre los casos anuales son los más citados en la literatura, Alvar et al. (2012) concluye que solo dos tercios de los países endémicos mantienen un registro de casos en un periodo de cinco años, por lo que se considera que el número de casos oficialmente reconocido es una sub-estimación. El agente etiológico de las leishmaniasis son varias especies de parásitos intracelulares del género Leishmania, cuya transmisión de humanos o reservorios vertebrados se realiza por la picadura de flebotomineos hembras infectados (KillickKendrick 1999). Los parásitos Leishmania spp. son mantenidos en la naturaleza por medio de huéspedes que en su mayoría son varios órdenes de mamíferos (Ashford 1996).

En México, se han reportado aproximadamente 21.833 casos (en promedio 1.162 casos anuales) durante el periodo de 1990-2020. Actualmente, son cuatro los estados que reportan $\approx 87 \%$ de los casos: Tabasco $(33,5 \%)$, Quintana Roo $(30,6 \%)$, Campeche $(12,8 \%)$ y Chiapas (9,8\%) (CENAPRECE 2020). Aunque existen varios reportes en la Península de Yucatán (PY) acerca de infecciones con Leishmania braziliensis Vianna (Canto-Lara et al. 1998), en la mayoría de los casos humanos estudiados, el principal agente causal es el parásito intracelular Leishmania mexicana (Biagi) (Biagi 1953). Los reservorios vertebrados de L. mexicana son: Peromyscus yucatanicus (J.A. Allen y Chapman) y Ototylomys phyllotis (Merriam), aunque existen otras especies infectadas naturalmente como Sigmodon hispidus (Say y Ord) y Oryzomys melanotis melanotis (Thomas) (Pech-May et al. 2016). Se sabe experimentalmente que al menos P. yucatanicus yucatanicus, puede mantener al parásito L. mexicana a lo largo de varios meses (Van Wynsbergue et al. 2000).

La leishmaniasis cutánea localizada es endémica en la PY (Seidelin 1912) y a partir de los trabajos pioneros de Shattuck (1938), Beltrán y Bustamante (1942), Biagi (1953), y Biagi y de Buen de Biagi (1953a, 1953b), se sabe que la zona de leishmaniasis corresponde a la selva mediana de la PY. En aquella época la distribución y extensión de la selva tropical correspondía con la zona económica donde se realizaban la extracción de los árboles de goma (Manilkara zapota (Royen)) Pennington y Sarukhán (2005). Actualmente, se desconocen las consecuencias a mediano y largo plazo de una alteración del ciclo enzoótico del parásito de leishmaniasis. Por lo que se requieren estudios ecoepidemiológicos sistemáticos y frecuentes que ayuden a la interpretación y cuantificación de los patrones de transmisión. Los estudios sobre incriminación de vectores en México 
datan del grupo de investigación de Francisco Biagi Filizola quienes demostraron que Bichromomyia olmeca olmeca [= Lutzomyia olmeca olmeca (Vargas y Díaz-Nájera)] era capaz de transmitir horizontalmente (=biológicamente) a L. mexicana (Biagi et al. 1965). En la PY -aparte de Bi. olmeca olmeca- se postula que tal vez otras especies como Lutzomyia cruciata (Coquillett), Psychodopygus panamensis [= Lutzomyia panamensis (Shannon)], Psathyromyia shannoni [= Lutzomyia shannoni (Dyar)] actúen también como vectores (Pech-May et al. 2016).

En México y en especial en la PY, la etapa de investigación sobre diversidad de especies e incriminación de vectores está aún vigente, pero existen todavía áreas dentro de la zona endémica que no han sido estudiadas en cuanto a vectores, reservorios y/o parásitos, como son Oaxaca (365 casos) o Nayarit (1.810 casos) (CENAPRECE 2020). En México, se han registrado hasta ahora 49 especies de flebotomineos y dos especies fósiles (Ibáñez-Bernal et al. 2015; Pech-May et al. 2016; Rodríguez-Rojas y Rebollar-Téllez 2017) siendo la región selvática neotropical de mayor riqueza y diversidad (Moo-Llanes et al. 2013). El estado de Campeche y en particular el municipio de Calakmul es un área endémica con transmisión activa de leishmaniasis cutánea localizada (Hernández-Rivera et al. 2015). En Campeche hasta ahora se han reportado 21 especies de flebotomineos, lo cual representa el 43,8 \% de las especies conocidas en el país (Biagi 1953; Rebollar-Téllez et al. 2005; Moo-Llanes et al. 2013). Debido a que muchos de los registros en Campeche han sido esporádicos y por la importancia de conocer la diversidad, riqueza de especies y estructura de las comunidades de flebotomineos en la región, es necesario realizar un análisis que permitan tener una visión de cómo está estructurada la biodiversidad en el municipio de Calakmul ya que estos datos pueden permitir obtener estimadores que sirvan como punto de referencia para estudios futuros. El objetivo de este estudio fue evaluar la diversidad y co-ocurrencia de los flebotomineos en siete localidades del municipio de Calakmul, Campeche, México.

\section{Materiales y Métodos}

Muestreo y recolectas. La recolecta de flebotomineos se realizó entre 2001-2007. En cada estación de trampeo se realizó un muestreo sistemático en dos localidades diferentes espacialmente. El muestreo se realizó en la época de mayor abundancia de flebotomineos (Rebollar-Téllez et al. 1996a; Andrade-Narváez et al. 2003), empleando trampas de luz CDC (Sudia y Chamberlain 1962), trampas cebadas Shannon (Shannon 1939) siguiendo lo recomendado por Pérez et al. (1987), y trampas Disney cebadas con Mus musculus Linnaeus (Disney 1966). Las trampas Shannon fueron cebadas con voluntarios hombres entre 21-35 años.

Las recolectas se realizaron a una distancia de $1,5 \mathrm{~km}$ del poblado. En cada sitio de muestreo se establecieron transectos lineales y cada 20-25 m de distancia se colocó un par de trampas a cada lado del transecto $(C D C=5 ;$ y Disney=5) y cada noche de colecta realizó una aleatorización (CDC y Disney) y (Shannon, $\mathrm{N}=1$ ). Las trampas CDC y Disney fueron colocadas a las $1800 \mathrm{~h}$ a $0700 \mathrm{~h}$, mientras la trampa Shannon de 1800 a $2200 \mathrm{~h}$. Los insectos fueron preservados en etanol al $70 \%$.

Sitios de Muestreo. Los sitios de muestreo se seleccionaron por su historial de incidencia de Leishmaniasis (Dr. Adelaido Chiñas, Departamento de Salud, H. Ayuntamiento del Municipio de Calakmul, comunicación personal 17 Julio 2000) y en función del acceso y autorización ejidal. Estas localidades fueron: Unión 20 de Junio (U20J), La Guadalupe (LG), La Virgencita (LV), Once de Mayo (OM), Dos Naciones (DN), Dos Lagunas Sur (DLS) y Arroyo Negro (AN). Tres localidades se encuentran dentro de la Reserva de la Biosfera de Calakmul (LG, DN y DLS) (Fig. 1). 


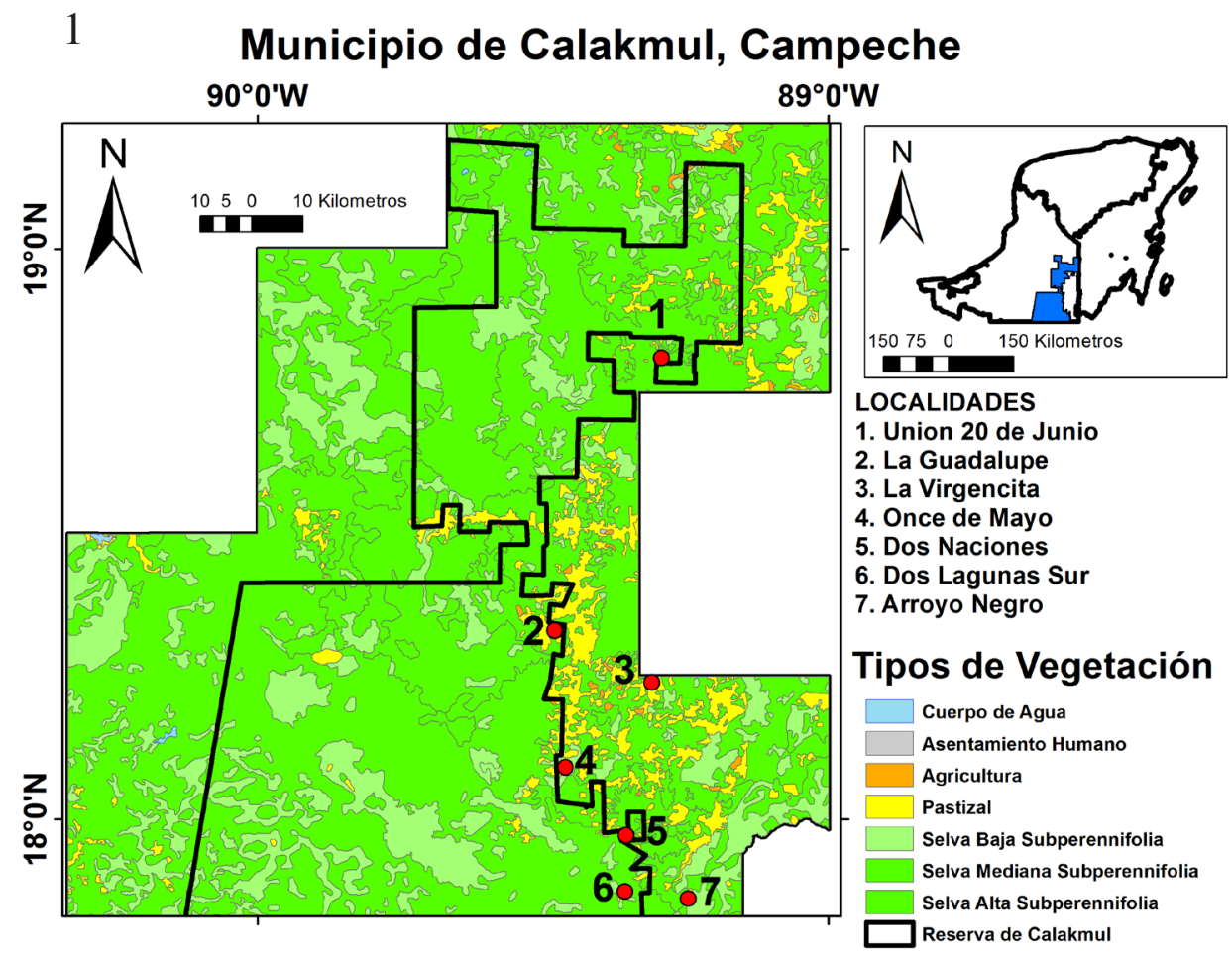

Figura 1. Mapa del municipio de Calakmul y el área correspondiente a la Reserva de la Biósfera delimitada por líneas en negro. Los diferentes colores representan los diferentes tipos de vegetación. El recuadro superior derecho señala la ubicación de la Península de Yucatán. Los sitios de estudio sistemáticos de flebotomineos en Calakmul, Campeche realizadas con diferentes tipos de trampas en el periodo de noviembre a marzo entre los años 2001-2007 se representan por puntos en color rojo (Ilustración por David A. Moo-Llanes).

Montaje e identificación. Los insectos fueron preparados para su identificación con la técnica de Ibáñez-Bernal (2000), que consistía primero en el deshidratado, los insectos se colocan en una placa de cerámica de tres pozos, posteriormente en esencia de clavo para su diafanización, y finalmente, se montan en resina de Euparal (Bioquip Products Inc, Rancho Grande, CA). La identificación de flebotomineos adultos se realizó con las claves de Ibáñez-Bernal (2005a, 2005b), Young (1979) y Young y Duncan (1994). Los especímenes mejor preparados se conservan en la colección del laboratorio de entomología médica de la Facultad de Ciencias Biológicas de la Universidad Autónoma de Nuevo León. La nomenclatura de las especies de flebotomineos sigue la clasificación de Galati $(1995,2003)$ (se incluye entre paréntesis el equivalente según el esquema tradicional), la cual en los años recientes ha sido más ampliamente aceptada que la tradicional basada en la propuesta de Lewis et al. (1977); mientras que la abreviación de géneros usa la propuesta de BrisolaMarcondes (2007).

Análisis de abundancia, riqueza, diversidad y co-ocurrencia. La estructura de las comunidades se analizó mediante los índices de diversidad alfa usando dos softwares: Species Diversity \& Richness (versión 3.03), y PAST (versión 2.16). No todos los índices fueron calculados con todos los programas, se usaron los que proveyeron la facilidad de cálculos y/o calidad de gráficos. Se obtuvo el índice de diversidad Alfa global de las localidades ubicadas dentro y fuera de la Reserva de la Biosfera de Calakmul. La riqueza específica se calculó con el índice de Margalef (DMg) (Margalef 1958); el cual permite estimar 
la biodiversidad en base a la distribución numérica de los individuos de las diferentes especies en función del número de individuos existentes en la muestra analizada (Moreno 2001), y el índice Alfa de Fisher (Fisher et al. 1943), que predice que muchas especies tienen frecuencias relativamente bajas y pocas especies resultan ser muy comunes y, por lo tanto, la distribución se ajusta a una serie logarítmica.

Para cuantificar la dominancia se utilizaron los índices de Simpson-Yule (Simpson 1949), el cual cuantifica la probabilidad de que un segundo individuo extraído de la población sea de la misma especie que el primero. Se calculó el índice de BergerParker, que cuantifica la proporción correspondiente a la especie dominante (Berger y Parker 1970). La heterogeneidad de la comunidad se estimó mediante el índice de Shannon-Weiner (H) (Shannon 1948). Este índice asume que los individuos son aleatoriamente muestreados y que todas las especies están contenidas en la muestra de una población infinitamente grande. Se calculó el estadístico Q (Kempton y Taylor 1978), que es una estimación de las abundancias de las especies y que considera solo la distancia intercuartil (Q3 y Q1), con lo que se elimina el sesgo ocasionado con las especies muy abundantes o raras. El software Species Diversity \& Richness se utilizó para calcular los siguientes índices: DMg, índice de Berger-Parker, H, y estadístico Q; mientras que el software PAST se usó para calcular el índice Alfa de Fisher y el índice Simpson-Yule.

Debido a que las abundancias de las especies pueden variar en función del sitio de estudio; se calculó el índice SISA (Standardized Index of Species Abundance), el cual es una estandarización por rangos en relación con la abundancia de cada especie por los sitios de estudio; y de esta manera poder hacer comparaciones directas entre sitios. De acuerdo con el índice SISA, las especies más comunes en todos los sitios tendrán los valores más altos de los rangos (Roberts y His 1979).

Para estimar el número efectivo de especies o "la diversidad verdadera", se calculó la fórmula propuesta por Jost (Jost 2006, 2007), cuyos resultados pueden expresarse como qD, donde la letra superescrita q indica el orden de la diversidad; por ejemplo $0 \mathrm{D}$ es la diversidad de orden cero o en otras palabras la riqueza específica, mientras que $1 \mathrm{D}$ es la diversidad de primer orden y resulta de la conversión de $\exp \left(\mathrm{H}^{\prime}\right)$ y con ello se obtiene el número efectivo de especies de una comunidad integrada por i especies igualmente comunes y 2D es la diversidad de segundo orden, la cual se basa más en las abundancias de las especies dominantes y no toma en consideración las especies raras (Moreno et al. 2001). Se realizó un análisis de la acumulación de especies en función del esfuerzo de muestreo; mediante la ecuación de Clench (Clench 1979), la cual es una función sigmoidal que predice la eficiencia de captura dado la asíntota de la curva. Para la curva de la acumulación de especies $\left[S(t)=a{ }^{*} t /\left(1+b^{*} t\right)\right]$, se realizó una aleatorización de 100 repeticiones con los softwares: EstimateS (versión 8.2.0) y Statistica (versión 10.0) (Jiménez y Hortal 2003).

La diversidad beta se determinó usando el estimador de Chao-Jaccard usando el software EstimateS versión 8.2.0. La co-ocurrencia de especies entre localidades se analizó usando modelos nulos con el índice C-Score (Stone y Roberts 1990). El índice C-Score (checkerboard score), fue utilizado para evaluar la distribución no aleatoria de los ensambles de especies. Los datos están basados en una matriz binomial de presencia (1) o ausencia (0) de especies se analizaron con ECOSIM (versión 7.72) y previo al análisis se realizaron 5.000 aleatorizaciones de los datos. De acuerdo con el modelo C-Score si el valor observado es mayor que el valor de C-Score de las simulaciones aleatorias, entonces se establece una co-ocurrencia no agregada. Por el contrario, si el valor observado es menor que C-Score de las simulaciones aleatorias se establece una co-ocurrencia agregada, y finalmente si los valores observados y simulados son iguales, se establece una co-ocurrencia aleatoria (Chaves 2011). 


\section{Resultados}

Durante 2001-2007 en el municipio de Calakmul se recolectaron 17.405 flebotomineos representados por nueve géneros y 17 especies, lo cual representó el 35,4\% de todas las especies en México, el 68,0\% de las especies conocidas en la PY y el 80,9\% de las especies reportadas para Campeche (Tabla 1). Se reportan dos nuevos registros para Campeche: Micropygomyia (Micropygomyia) cayennensis maciasi [= L. cayennensis maciasi] (LV)] y Dampfomyia (Coromyia) steatopyga [= L. steatopyga (U20J y DLS)]. La abundancia y porcentaje de flebotomineos por localidad fue: U20J (1.743 ejemplares; 10,0\%), LG $(4.118 ; 23,7 \%)$, LV (2 623; 15,1\%), OM (3.135; 18,0\%), DN (4.340; 24,9\%), DLS (1.245; 7,2\%) y AN (3.135; 1,2\%). Las localidades que presentaron el menor número de especies fueron OM y AN con ocho especies, mientras que la localidad que presentó el mayor número de especies fue DN con 14. Cinco especies de flebotomineos representaron aproximadamente el $88,8 \%$ de toda la colecta siendo éstas consideradas como especies comunes: Bi. olmeca olmeca (22,2\%), Lu. cruciata (19,3\%), Ps. panamensis (18,8\%), Pa. shannoni (14,8\%) y Dampfomyia deleoni (13,7\%), reportándose en todas las localidades. También, Psathyromyia carpenteri, se presentó en todas las localidades, aunque en menor abundancia (2,4\%). Otras especies fueron consideradas comunes, por ejemplo, Pintomyia ovallesi (4,5\%, en cinco localidades), Brumptomyia mesai $(1,8 \%$, en seis localidades) y Micropygomyia trinidadensis ( $0,7 \%$, en cinco localidades). Por el contrario, tres especies, se colectaron solamente en una localidad: Dampfomyia permira (U20J), Lutzomyia gomezi (DN) y Mi. cayennensis maciasi (LV). Sin embargo, existen especies que se presentaron en dos o tres localidades, pero la mayor abundancia se localiza en una localidad: Brumptomyia hamata (95\%, DN), Da. steatopyga (92,9\%, U20J), Nyssomyia ylephiletor $(75,9 \%, \mathrm{DN})$ y Psathryomyia undulata $(97,9 \%, \mathrm{OM})$ (Tabla 1). El índice de SISA mostró que Bi. olmeca olmeca $(2,14)$, Ps. panamensis $(2,86)$, Da. deleoni $(3,43)$, y Pa. shannoni $(4,0)$ tuvieron los mayores valores (Tabla 1$)$.

Tabla 1. Abundancia de flebotomineos de Calakmul, Campeche, México, durante 2001-2007. U20J= Unión 20 de Junio; LG= La Guadalupe; LV= La Virgencita; OM= Once de Mayo; DN= Dos Naciones; DLS= Dos Lagunas Sur; AN= Arroyo Negro. SISA= Standarized Index of Species Abundances.

\begin{tabular}{|c|c|c|c|c|c|c|c|c|c|}
\hline \multirow[t]{2}{*}{ Especies } & \multicolumn{7}{|c|}{ LOCALIDADES DE CALAKMUL } & \multirow[t]{2}{*}{ SISA } & \multirow[t]{2}{*}{ Total } \\
\hline & U20J & LG & LV & $\mathrm{OM}$ & $\mathrm{DN}$ & DLS & AN & & \\
\hline Bichromomyia o. olmeca & 369 & 1044 & 1230 & 553 & 352 & 277 & 36 & 0,92 & 3.861 \\
\hline Brumptomyia mesai & 32 & 8 & 5 & 0 & 232 & 28 & 1 & 0,42 & 306 \\
\hline Brumptomyia hamata & 0 & 0 & 0 & 0 & 19 & 1 & 0 & 0,07 & 20 \\
\hline Dampfomyia deleoni & 346 & 658 & 638 & 42 & 440 & 233 & 25 & 0,82 & 2.382 \\
\hline Dampfomyia permira & 1 & 0 & 0 & 0 & 0 & 0 & 0 & 0,05 & 1 \\
\hline Dampfomyia steatopyga & 13 & 0 & 0 & 0 & 0 & 1 & 0 & 0,09 & 14 \\
\hline Lutzomyia cruciata & 301 & 1822 & 146 & 602 & 332 & 78 & 84 & 0,83 & 3.365 \\
\hline Lutzomyia gomezi & 0 & 0 & 0 & 0 & 3 & 0 & 0 & 0,01 & 3 \\
\hline Lutzomyia longipalpis & 0 & 0 & 10 & 2 & 3 & 0 & 1 & 0,22 & 16 \\
\hline Micropygomyia c. maciasi & 0 & 0 & 1 & 0 & 0 & 0 & 0 & 0,04 & 1 \\
\hline Micropygomyia trinidadensis & 1 & 6 & 6 & 0 & 100 & 8 & 0 & 0,28 & 121 \\
\hline Nyssomyia ylephiletor & 0 & 0 & 0 & 0 & 60 & 19 & 0 & 0,12 & 79 \\
\hline Pintomyia ovallesi & 36 & 114 & 85 & 0 & 504 & 42 & 0 & 0,52 & 781 \\
\hline Psathyromyia carpenteri & 15 & 23 & 62 & 8 & 301 & 3 & 5 & 0,52 & 417 \\
\hline
\end{tabular}




\begin{tabular}{|l|c|c|c|c|c|c|c|c|c|}
\hline Psathyromyia shannoni & 272 & 168 & 149 & 1516 & 310 & 137 & 29 & 0,78 & 2.581 \\
\hline Psathyromyia undulata & 0 & 1 & 0 & 185 & 3 & 0 & 0 & 0,16 & 189 \\
\hline Psychodopygus panamensis & 357 & 274 & 291 & 227 & 1,681 & 418 & 20 & 0,86 & 3.268 \\
\hline Número de especies & 11 & 10 & 11 & 8 & 14 & 12 & 8 & --- & 17 \\
\hline
\end{tabular}

El índice Margalef (DMg) considerando todas las localidades fue de 1,63 y por localidades los valores más altos fueron en (OM y DN). El índice alfa de Fisher para todas las localidades fue de 1,85 y las localidades con los valores más altos fueron OM y DN. La dominancia con el índice de Simpson-Yule para todas las localidades fue de 0,16 , y los valores más altos en $\operatorname{U} 20 \mathrm{~J}(0,31)$, DLS $(0,29)$ y AN $(0,29)$. Una tendencia similar fue encontrada con el índice de Berger-Parker, que para todas las localidades resultó en 0,22 y los valores más altos en U20J $(0,48)$, AN $(0,46)$ y DLS $(0,44)$.

El índice de heterogeneidad Shannon-Weiner arrojó un valor general de 1,95 y en DN 1,96 y LV 1,80. Similar a lo obtenido con el estadístico Q, que para los datos combinados de todas las localidades dio 1,59 y los valores más altos fueron para DN $(2,39)$ y LG $(2,02)$. La diversidad verdadera expresada como de primer orden $1 \mathrm{D}$, para todas las localidades fue de 7,03 y los valores más altos fueron para DN $(7,10)$ y LV $(6,05)$ (Tabla 2).

Tabla 2. Diversidad Alfa de flebotomineos en Calakmul, Campeche, México en 2001-2007. U20J= Unión 20 de Junio; LG= La Guadalupe; LV= La Virgencita; $\mathrm{OM=}$ Once de Mayo; DN= Dos Naciones; DLS= Dos Lagunas Sur; AN= Arroyo Negro. Total= Incluye los datos de todas las localidades anteriores. Nota: Diversidad de orden $0=$ solamente es el número de especies. Diversidad de orden $1=$ Exponencial de índice de Shannon-Wiener. Diversidad de orden 2=1/ índice de Simpson.

\begin{tabular}{|l|c|c|c|c|c|c|c|c|}
\hline & \multicolumn{7}{|c|}{ SITIOS DE ESTUDIO } & \multirow{2}{*}{ Total } \\
\cline { 2 - 8 } & U20J & LG & LV & OM & DN & DLS & AN & \\
\hline Riqueza de especies & & & & & & & & \\
\hline Margalef & 0,87 & 1,32 & 1,34 & 1,54 & 1,52 & 1,08 & 1,27 & 1,63 \\
\hline Alfa de Fisher & 0,99 & 1,66 & 1,56 & 1,84 & 1,79 & 1,23 & 1,46 & 1,85 \\
\hline Dominancia & & & & & & & & \\
\hline Simpson-Yule & 0,31 & 0,25 & 0,18 & 0,21 & 0,20 & 0,29 & 0,29 & 0,16 \\
\hline Berger-Parker & 0,48 & 0,41 & 0,21 & 0,33 & 0,38 & 0,44 & 0,46 & 0,22 \\
\hline Heterogeneidad & & & & & & & & \\
\hline Shannon-Wiener & 1,40 & 1,58 & 1,80 & 1,75 & 1,96 & 1,46 & 1,51 & 1,95 \\
\hline Estadístico Q & 1,52 & 2,02 & 1,82 & 1,78 & 2,39 & 1,13 & 1,54 & 1,59 \\
\hline Diversidad Verdadera & & & & & & & & \\
\hline Diversidad de orden 0 & 11 & 10 & 11 & 8 & 14 & 12 & 8 & 17 \\
\hline Diversidad de orden 1 & 4,06 & 4,85 & 6,05 & 5,75 & 7,10 & 4,31 & 4,53 & 7,03 \\
\hline Diversidad de orden 2 & 3,23 & 4,00 & 5,56 & 4,76 & 5,00 & 3,45 & 3,45 & 6,25 \\
\hline
\end{tabular}

La diversidad beta Chao-Jaccard muestra que la mayor diversidad se presentó en U20J-LG, LG-LV y DN-DLS, las cuales comparten $99 \%$ de especies $(\approx 17)$. Se observa en la matriz de valores comparativos (Tabla 3); que las comunidades que menos especies compartidas fueron OM y DN (78\%). OM fue la localidad que presentó el menor número de especies que comparte con el resto de las localidades (Fig. 2). 


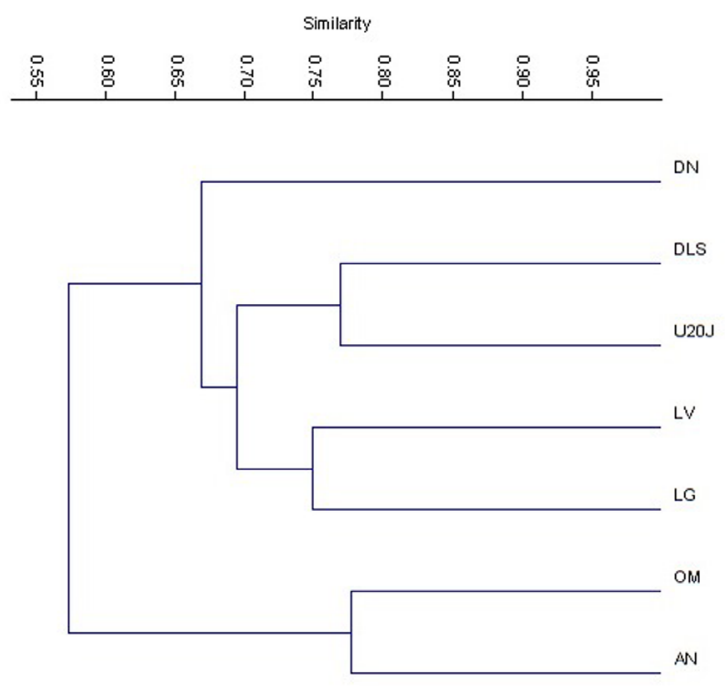

Figura 2. Dendograma de Diversidad Beta de Flebotomianos. Ilustrando la similitud en función del estimador de Chao-Jaccard.

La curva de acumulación de especies analizada mediante la ecuación de Clench, mostró que existe un buen ajuste entre los datos observados y la predicción de la acumulación de especies hasta alcanzar a asíntota de la curva. Los valores encontrados fueron de R2=0,9942; $a=28,47 ; b=1,60$. En función de los parámetros de la ecuación de Clench, se predice que el total de especies presentes es 18; con las 17 especies registradas en el estudio, se encuentra que esto representa 96\% de la riqueza total esperada en Calakmul (Fig. 3).

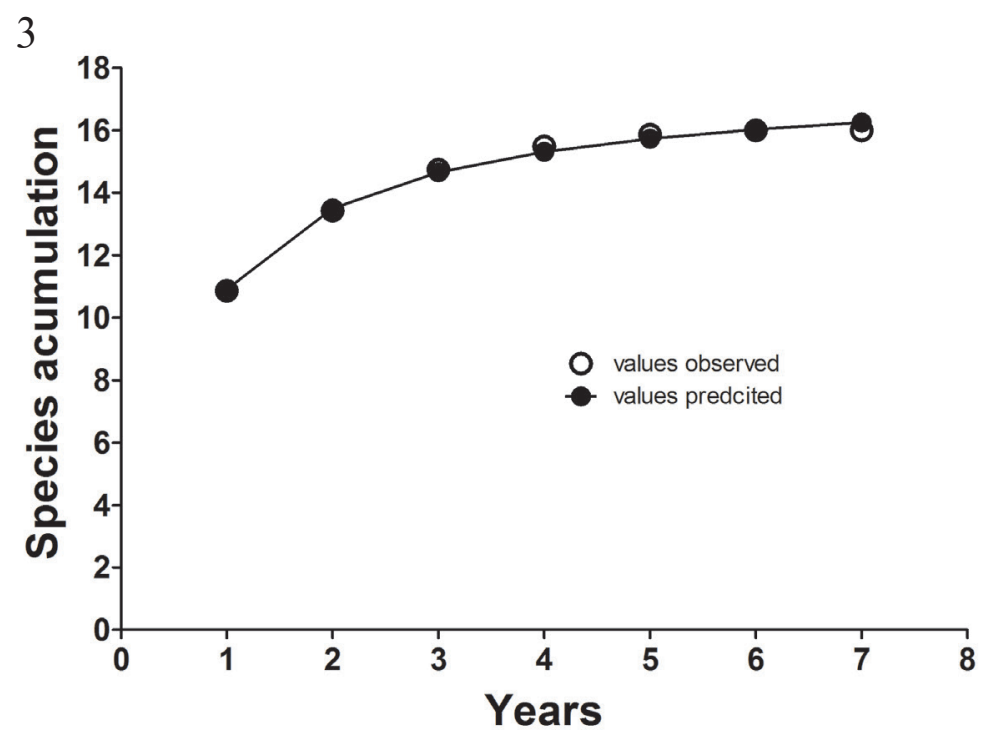

Figura 3. Curva de acumulación de flebotomineos usando el modelo de Clench (1979), en función del esfuerzo de muestreo (años).

El análisis de co-ocurrencia de especies usando el modelo de C-score dio un resultado de 0,573 , el cual no fue significativo $(p=0,447)$ al compararlo con el valor medio simulado 0,586 (varianza $=0.00117$ ), lo cual indica una co-occurrencia agregada espacial de especies entre las distintas localidades de Calakmul. 
Tabla 3. Diversidad Beta de Flebotomineos en Calakmul, Campeche, México en 2001-2007. U20J= Unión 20 de Junio; LG= La Guadalupe; LV= La Virgencita; OM= Once de Mayo; DN= Dos Naciones; DLS= Dos Lagunas Sur; AN= Arroyo Negro. *Mayor diversidad beta= especies compartidas entre localidades.

\begin{tabular}{|c|c|c|c|c|c|c|c|}
\hline \multicolumn{7}{|c|}{ LOCALIDADES DE CALAKMUL } \\
\hline & U20J & LG & LV & OM & DN & DLS & AN \\
\hline U20J & & & & & & & \\
\hline LG & $0,99^{*}$ & & & & & & \\
\hline LV & 0,98 & $0,99^{*}$ & & & & & \\
\hline OM & 0,89 & 0,96 & 0,90 & & & & \\
\hline DN & 0,97 & 0,98 & 0,98 & 0,78 & & & \\
\hline DLS & 0,98 & 0,98 & 0,97 & 0,87 & $0,99^{*}$ & & \\
\hline AN & 0,96 & 0,96 & 0,96 & 0,93 & 0,84 & 0,93 & \\
\hline
\end{tabular}

\section{Discusión}

En México, aún existe muchas áreas en el campo de los flebotomineos por investigar, como es el caso de la búsqueda de nuevas especies (Ibáñez-Bernal et al. 2006, 2010), estados sin registros como son Aguascalientes, Baja California Sur, Colima, Durango, Guanajuato, Querétaro, Tlaxcala y Zacatecas. En la actualidad, es muy escaso el conocimiento acerca del estudio de patrones de diversidad de flebotomineos en el país, de hecho, solo se tienen reportado dos trabajos (May-Uc et al. 2011; Rodríguez-Rojas y Rebollar-Téllez 2017) para el estado de Quintana Roo. Se reportaron dos nuevos registros para Campeche: Mi. cayennensis maciasi y Da. steatopyga. Por lo cual, se amplía el rango de distribución de estas especies, apoyando la distribución potencial para flebotomineos de Centro y Norteamérica (Moo-Llanes et al. 2013).

Las cinco especies de mayor abundancia en este estudio (Bi. olmeca olmeca, Lu. cruciata, Ps. panamensis, $\mathrm{Pa}$. shannoni y $\mathrm{Da}$. deleoni) se han considerado en diferentes estudios como las de mayor abundancia en toda la PY (Biagi et al. 1965; Rebollar-Téllez et al. 1996a, 1996b, 1996c; Pech-May et al. 2010). En los datos aquí presentados se encontró que estas cinco especies de flebotomineos resultaron con los valores más altos del índice SISA, lo cual indica que las especies son abundantes aun compensando por el efecto de la variable localidad. Resultados similares se encontraron en Limones, Balacar, Quintana Roo para estas cuatro especies (Bi. olmeca olmeca, Lu. cruciata, Pa. shannoni y Da. deleoni) (RodríguezRojas y Rebollar-Téllez 2017) En términos epidemiológicos es muy importante destacar estas cinco especies, a excepción de Da. deleoni - son consideradas con capacidad para actuar como vectores de Leishmania spp. en el sureste de México (Pech-May et al. 2010; Sánchez-García et al. 2010; Pech-May et al. 2016).

En algunas localidades como DN hubo cierta abundancia de especies no implicadas hasta ahora en la epidemiología de las leishmaniasis como Mi. trinidadensis y Br. hamata. No se puede concluir acerca de la causalidad de la abundancia de estas especies, pero es posible que los muestreos en DN hayan sido cerca de cuevas kársticas (Montes de OcaAguilar et al. 2013a) o madrigueras de mamíferos (Montes de Oca-Aguilar et al. 2013b), ya que estas especies han sido usualmente reportadas asociadas a estos ecotopos por su alta disponibilidad de alimentación.

Para monitorear el efecto de los cambios en el ambiente es necesario contar con la información de diversidad biológica en comunidades naturales y modificadas (diversidad alfa) y también la tasa de recambio de especies en la biodiversidad entre distintas 
comunidades (diversidad beta), o para conocer su contribución a nivel regional (diversidad gama) (Moreno 2001). Existen diversos estudios cuantitativos sobre diversidad alfa o beta, han sido publicados principalmente para biotas de Phlebotominae en Colombia (Porter y DeFoliart 1981), Costa Rica (Jiménez et al. 2000), Brasil (De Luca et al. 2003); Venezuela (Rojas et al. 2004), Paraguay (Salomón et al. 2003) y Argentina (Salomón et al. 2004). Interesantemente en algunos de esos estudios (Jiménez et al. 2000; Rojas et al. 2004) se ha reportado que cuando se han comparado biológicamente la estructura de las comunidades se ha encontrado que las diferencias en diversidad beta, obedecen más a cambios de las abundancias relativas, que ha cambios por la presencia de diferentes las especies.

En términos comparativos con el resto del país, la PY es considerada como un área de alta riqueza de especies y diversidad de flebotomineos. En Campeche, se han reportado a la fecha 21 especies, lo que representa el 43,8 \% de flebotomineos del país (Moo-Llanes et al. 2013; Pech-May et al. 2016). Los datos de la curva de acumulación de especies indica que el esfuerzo de captura fue óptimo para registrar un 96,10\% de 18 especies. Es posible que en Calakmul también estén presentes otras especies como: Dampfomyia dodgei, Psathyromyia aclydifera, Psathyromyia cratifer y Pintomyia serrana, las cuales se han registrado en el vecino municipio de Escárcega (Rebollar-Téllez et al. 1996a; Moo-Llanes et al. 2013).

Las localidades con mayor biodiversidad basado en el número de especies (riqueza específica), fueron (OM y DN), con lo que se puede suponer que ambas localidades son estructuralmente más ricas en especies. En relación con la dominancia, tres localidades (U20J, DLS y AN) tuvieron los mayores índices de dominancia, sin importar el índice utilizado, los cuales se asocian con su baja heterogeneidad. Las localidades LG, LV y DN tuvieron los mayores índices de heterogeneidad. Además, se encontró que existía una mayor abundancia e índices más altos de heterogeneidad en las localidades ubicadas dentro del área restringida a la Reserva de Calakmul (LG, DN y DLS). Estos datos son similares a los presentados en varios países de América, donde se ha encontrado una mayor abundancia en selvas en comparación a los bordes de éstas o en zonas con algún manejo productivo (Bejarano et al. 2002; Valderrama et al. 2008).

Es interesante notar que la diversidad verdadera según Jost (2006) expresada como de primer orden (1D), coincide con el índice de Shannon-Weiner y Q. Se podría concluir que las localidades de mayor diversidad son DN, LV y LG. También se observó que existe un alto grado de especies compartidas en estas localidades, lo que quizás se deba a que existe una carretera que cruza de en dirección sur-norte desde el Municipio de Hopelchén pasando por muchas de las localidades de Calakmul, siendo AN el último asentamiento humano, la cual podría servir para corredor biológica asistido por los habitantes en sus trayectos. El análisis de la estructura del ensamble y gremio de Phebotominae en México es aún incipiente. Aunque si bien es cierto, que el análisis de diversidad beta refleja un alto grado de especies compartidas, también se registraron diferencias en cuanto a la estructura de diversidad biótica entre los sitios de estudio. Interesantemente, el dendrograma generado entre las localidades (Fig. 7, Tabla 3), demuestra que la similitud entre sitios no obedece a un gradiente de cercanía por distancia entre ellos. Este patrón de distribución o diversidad beta, es aún incógnita en cuanto a las variables bióticas o abióticas que subyacen su estructura. Hasta el momento, solamente Rodríguez-Rojas et al. (2017) había evaluado en México, la co-ocurrencia de especies de flebotomineos usando el índice de C-score en la región de Nuevo León y Tamaulipas, obteniendo una co-ocurrencia agregada (RodríguezRojas et al. 2017). En Venezuela, Carzola et al. (2014) encontró una co-ocurrencia aleatoria en 10 localidades usando diferentes técnicas de captura; igualmente una co-ocurrencia agregada para especies solamente antropofílicas y una co-ocurrencia no agregada para especies zoofílicas. Al comparar los estudios con una coocurrencia agregada, resaltan especies compartidas: Br. hamata, Br. mesai, Lu. cruciata, Lu. gomezi, Lu. longipalpis y Ps. shannoni, las cuales podrían estar jugando un importante rol en el ensamble de especies y 
la co-ocurrencia agregada en México.

Finalmente, existen nuevos registros para el estado. Es importante mencionar, que los estimadores de biodiversidad son efectivos para explicar la riqueza y estructura de la comunidad, se argumenta la utilidad del estadístico $Q$, que, al no ser influenciado por especies abundantes o especies raras, resultaría en una buena estimación de la heterogeneidad. Existen muchas especies de flebotomineos compartidos entre la mayoría de las localidades, lo cual es un punto crucial para poder establecer medidas de control vectorial o de prevención a la infección, al ser considerado el estado como un área endémica de transmisión de la leishmaniasis. El esquema de los estudios de biodiversidad como el aquí reportado, podría servir de base para futuros estudios en otras localidades con prevalencia de infección por Leishmania spp. Se propone igualmente que este tipo de estudios se debe de complementar con análisis cuantitativos de redes de interacción ecológica con las especies de mamíferos y otras especies de otros gremios similares presentes en las mismas zonas y con ello poder elucidar las relaciones de patrones de alimentación como un proxy a las preferencias por selección del hospedero y que con ello se pueda entender mejor la compleja red ecológica en términos de la transmisión del parásito Leishmania spp.

\section{Agradecimientos}

Esta investigación fue realizada en el Centro de Investigaciones Regionales "Dr. Hideyo Noguchi" de la Universidad Autónoma de Yucatán. Se agradece la valiosa participación de los ejidatarios de todas las comunidades en Calakmul, Campeche. También se agradece la colaboración del médico Adelaido Chiñas por haber facilitado la comunicación con los líderes comunitarios de los ejidos mientas él laboraba en la clínica rural del IMSS en el ejido Ing. Ricardo Payró Jene ("Polo Norte") y luego en el departamento de Salud H. Ayuntamiento del Municipio de Calakmul. Se reconoce la muy entusiasta participación en campo y laboratorio de los biólogos Ezequiel Tun Ku, Felipe A. Dzul Manzanilla, Azael Che Mendoza, Rurik Barroso Burgos, Gabriel Peraza Herrera, Miguel A. Palma Rufino y Concepción Méndez Pérez, a todos ellos se les agradece por soportar las inclemencias del tiempo y todas las vicisitudes vividas para coordinar y llevar acabo el trabajo de campo. Gracias a todo ello; tenemos interesantes y -hasta cierto punto - jocosas anécdotas compartidas.

Esta investigación fue financiada por Programa de Entrenamiento e Investigación en Enfermedades Tropicales de la Organización Mundial de la Salud (UNDP/WHO/TDR) ID A00732 y por el proyecto: Capacidad y competencia vectorial de Lutzomyia cruciata. Implicaciones en la transmisión de Leishmania mexicana (CONACYT 2004-C01-47194), otorgados a EART.

Contribución de los Autores. Rebollar-Téllez, E.A. (Conceptualización, Investigación, Escritura, Revisión y Edición del Manuscrito). Moo-Llanes, D.A. (Conceptualización, Análisis de datos, Investigación, Escritura, Revisión y Edición del Manuscrito).

Conflictos de interés. No existen conflictos de interés entre los investigadores.

\section{Literatura Citada}

Alvar, J., Vélez, I.D., Bern, C., Herrero, M., Desjeux, P., Cano, J., Jannin, J., den Boer, M., The WHO Leishmaniasis Control Team (2012) Leishmaniasis worldwide and global estimates of its incidence. PLoS ONE, 7: e35671.

Andrade-Narváez, F., Canto-Lara, S., Van Wynsbergue, N., Rebollar-Téllez, E.A., Vargas, A. y Albertos, N. (2003) Seasonal transmission of Leishmania (Leishmania) mexicana in the state of Campeche, Yucatán Peninsula. Memorias do Instituto Oswaldo Cruz, 98: 995-998. 
Ashford, R. (1996) Leishmaniasis reservoirs and their significance in control. Clinics in Dermatology, 14: 523-532.

Ashford, R.W., Desjeux, P. y de Raadt, P. (1992) Estimation of population at risk of infection and number of cases of leishmaniasis. Parasitology Today, 8: 104-105.

Bejarano, E., Uribe, S., Rojas, W. y Vélez, ID. (2002) Phlebotomine sand flies (Diptera: Psychodidae) associated with the appearance of urban Leishmaniasis in the city of Sincelejo, Colombia. Memorias do Instituto Oswaldo Cruz, 97: 645-647.

Beltrán, E. y Bustamante, M.E. (1942) Datos epidemiológicos acerca de la "úlcera de los chicleros" (Leishmaniasis americana) en México. Revista del Instituto de Salubridad y Enfermedades Tropicales, 3: 1-28.

Berger, W.H. y Parker, F.L. (1970) Diversity of planktonic foraminifera in deep sea sediments. Science, 168: 1345-1347.

Biagi, F.F., de Buen de Biagi, A.M. y Beltrán, F. (1965) Phlebotomus flaviscutellatus transmisor natural de Leishmania mexicana. Prensa Médica México, 30: 267-272.

Biagi, F.F.y de Buen de Biagi,A.M. (1953a) Algunosflebotomus delárea endémica deleishmaniasis tegumentaria americana del estado de Campeche, México. Medicina, 33: 315-319.

Biagi, F.F. y de Buen de Biagi, A.M. (1953b) Datos ecológicos de algunos flebotomus mexicanos (Diptera, Psych.). Anales del Instituto de Biología de México, 24: 445-450.

Biagi, F.F. (1953) Algunos comentarios sobre las leishmaniasis y sus agentes etiológicos. Leishmania tropica mexicana, nueva subespecie. Medicina, 33: 401-406.

Brisola-Marcondes, C. (2007) A proposal of generic and subgeneric abbreviations for phlebotomine sandflies (Diptera: Psychodidae: Phlebotominae) of the world. Entomological News, 118: 351-356.

Canto-Lara, S.B., Cárdenas-Marrufo, M.F., Vargas-González, A. y Andrade-Narváez, A.F. (1998) Isoenzyme characterization of Leishmania isolated from human cases with localized cutaneous leishmaniasis from the state of Campeche, Yucatan Peninsula, Mexico. American Journal of Tropical Medicine and Hygiene, 58: 444-447.

Carzorla, D.J., Nieves, E. y Morales, P. (2014) Patrones de coocurrencia y conducta alimenticia a escala local de Phlebotominae (Diptera: Psychodidae) del estado Falcón, Venezuela. Revista Peruana de Biología, 21: 99-104.

Centro Nacional de Programas Preventivos y Control de Enfermedades (CENAPRECE) (2020) Boletín epidemiológico anual 1990-2020. Disponible en www.dgepi.salud.gob.mx.

Chaves, L.F. (2011) Phlebotomine sand fly species co-occurrence at the local scale: differences between agricultural and forested areas. Boletin de Malariología de Salud Ambiental, 51: 3539.

Clench, H. (1979) How to make regional lists of butterflies: some thoughts. The Journal of the Lepidopterists' Society, 33: 216-231.

De Luca, A.S., Vasconcelos, H.L. y Barrett, T.V. (2003) Distribution of sandflies (Diptera: Phlebotominae) in forest remmants and adjacent matrix habitats in Brazilian Amazonia. Brazilian Journal of Biology, 63: 401-410.

Disney, R. (1966) Observations on zoonosis: Leishmaniasis in British Honduras. Journal of Applied Ecology, 5: 1-59.

Fisher, R.A., Corbert, A.S. y Williams, C.B. (1943) The relation between the number of species and the number of individuals in a random sample of an animal population. Journal of Animal Ecology, 12: 42-58.

Galati, E.A.B. (2003) Classificação de Phlebotominae, 2003; pp. 23-51. In: Rangel E.F. y R. Lainson (eds.) Flebotomíneos do Brasil. Rio de Janeiro: Fiocruz.

Galati, E.A.B. (1995) Phylogenetic systematics of Phlebotominae (Diptera, Psychodidae) with emphasis on American groups. Boletin de la dirección de Malariología y Salud Ambiental, 1: 133-142. 
Hernández-Rivera, M.P., Hernández-Montes, O., Chiñas-Pérez, A., Batiza-Avelar, J.B., Sánchez-Tejeda, G.S., Wong-Ramírez, C. y Monroy-Ostria, A. (2015) Study of cutaneous leishmaniasis in the state of Campeche (Yucatan Peninsula), Mexico, over a period of two years. Salud Pública de México, 57: 58-65.

Ibáñez-Bernal, S., Hernández-Xoliot, R.A. y Mendoza, F. (2006) Collections of Bruchomyiinae and Phlebotominae (Diptera: Psychodidae) from the north-central portion of the state of Veracruz, México, with the description of a new species. Zootaxa, 1270: 19-33.

Ibáñez-Bernal, S., May-Uc, E. y Rebollar-Téllez, E.A. (2010) Two new species of phlebotomine sand flies (Diptera: Psychodidae, Phlebotominae) from Quintana Roo, Mexico. Zootaxa, 2448: 26-34.

Ibáñez-Bernal, S., Muñoz, J., Rebollar-Téllez, E.A., Pech-May, A. y Marina, C. (2015) Phlebotomine sand flies (Diptera: Psychodidae) of Chiapas collected near the Guatemala border, with additions to the fauna of Mexico and a new subgenus name. Zootaxa, 3994: 151-186.

Ibáñez-Bernal, S. (2005a) Phlebotominae (Diptera: Psychodidae) de México. V. Clave ilustrada para la identificación de los machos de Lutzomyia Franca. Folia Entomológica Mexicana, 44: 49-66.

Ibáñez-Bernal, S. (2005b) Phlebotominae (Diptera: Psychodidae) de México. VI. Clave ilustrada para la identificación de las hembras de Lutzomyia Franca. Folia Entomológica Mexicana, 44: 195-212.

Ibáñez-Bernal, S. (2000) Psychodidae(Diptera). In: Llorente, J., E. González y Papavero, N. (Eds). 607-626 pp. Biodiversidad, Taxonomía y Biogeografía de artrópodos de México. Hacia una síntesis de su conocimiento. Vol. II. México, D.F. Universidad nacional Autónoma de México. ISBN: 968-36-8003-8.

Jiménez, A. y Hortal, J. (2003) Las curvas de acumulación de especies y la necesidad de evaluar la calidad de los inventarios biológicos. Revista Iberoamericana de Aracnología, 8: 151-161.

Jiménez, A.E., Rojas, J.C., Vargas, F. y Herrero, M.V. (2000) Temporal and spatial variation of phlebotomine (Diptera: Psychodidae) community diversity in a cutaneous leishmaniasis endemic area of Costa Rica. Journal of Medical Entomology, 37: 216-221.

Jost, L. (2006) Entropy and diversity. Oikos, 113: 363-375.

Jost, L. (2007) Partioning diversity into independent alpha and beta components. Ecology, 88: 2427-2439.

Kempton, R.A. y Taylor, R.L. (1978) The Q-statistic and the diversity of floras. Nature, 275: 252-253.

Killick-Kendrick, R. (1999) The biology and control of phlebotominae sand flies. Clinics in Dermatology, 17: 279-289.

Lewis, D., Young, D., Minter, D. y Fairchild, G. (1977) Proposals for stable classification on the phlebotomine sandflies (Diptera: Psychodidae). Systematic Entomology, 2: 319-332.

Margalef, D.R. (1958) Information theory in ecology. General Systems, 3: 36-71.

May-Uc, E., Hernández, H. y Rebollar-Téllez, E.A. (2011) Distribución de flebotomineos (Diptera: Psychodidae) en Quintana Roo, México. Acta Zoológica Mexicana, 27: 273-289.

Montes de Oca-Aguilar, A.C., Moo-Llanes, D.A. y Rebollar-Téllez, E.A. (2013a) Sand fly species from a karstic cave in the Península of Yucatan, Mexico. Entomological News, 123: 191-200.

Montes de Oca-Aguilar, A.C., Moo-Llanes, D.A. y Rebollar-Téllez, E.A. (2013b) Adults sand fly species from diurnal resting sites on the Peninsula of Yucatán, México. Southwestern Entomologist, 38: 241-250. 
Moo-Llanes, D.A., Ibarra-Cerdeña, C.N., Rebollar-Téllez, E.A., Ibáñez-Bernal, S., González, C. y Ramsey, J.M. (2013) Current and future niche of North and Central American sand flies (Diptera: Psychodidae) in climate change scenarios. PLoS Neglected Tropical Disease, 7: e2421.

Moreno, C. (2001) Métodos para medir la Biodiversidad. MyT-Manuales y Tesis, Sociedad de Entomología Aragonesa (SEA). Vol. 1. Zaragoza, España. 84 pp.

Moreno, C.E., Barragán, F., Pineda, E. y Pavón, N.P. (2011) Reanálisis de la diversidad alfa: alternativas para interpretar y comparar información sobre comunidades ecológicas. Revista Mexicana de Biodiversidad, 82: 1249-1261.

Pech-May, A., Escobedo-Ortegón, F.J., Berzunza-Cruz, M. y Rebollar-Téllez, E.A. (2010) Incrimination of four sand fly previously unrecognized as vectors of Leishmania parasites in Mexico. Medical and Veterinary Entomology, 24: 150-161.

Pech-May, A., Peraza-Herrera, G., Moo-Llanes, D.A., Escobedo-Ortegón, F.J., Berzunza-Cruz, M., Becker-Fauser, I., Montes de Oca-Aguilar, A.C. y RebollarTéllez, E.A. (2016) Assessing the importance of four sandfly species (Diptera: Psychodidae) as vectors of Leishmania mexicana in Campeche, Mexico. Medical and Veterinary Entomology, 30: 310-320.

Pennington, T.D. y Sarukhán, J. (2005) Árboles Tropicales de México. Manual para la Identificación de las Principales Especies. Texto Científico Universitario. Dirección General de Publicaciones y Fomento Editorial UNAM, Fondo de Cultura Económica, México, D. F. 523 pp.

Pérez, J.E., Villaseca, P., Llanos-Cuentas, A., Campos, M. y Guerra, H. (1987) Técnicas para colectar "titiras" (Lutzomyia spp., Diptera: Psychodidae) en ambientes altoandinos peruanos. Revista Peruana de Entomología, 30: 77-80.

Porter, C.H. y DeFoliart, G.R. (1981) The man-biting activities of phlebotomine sand flies (Diptera: Psychodidae) in a tropical wet forest environment in Colombia. Arquivos de Zoologia Universidade de São Paulo, 30: 81-158.

Rebollar-Téllez, E.A., Ramírez, A. y Andrade, F. (1996a) A two years study on vectors of cutaneous leishmaniasis. Evidence for sylvatic transmission cycle in the state of Campeche, Mexico. Memorias do Instituto Oswaldo Cruz, 91: 555-560.

Rebollar-Téllez, E.A., Reyes, F., Fernández-Salas, I. y Andrade, F. (1996b) Population dynamics and biting rhythm of the anthropophilic sandfly Lutzomyia cruciata (Diptera: Psychodidae) in Southeast, Mexico. Revista del Instituto de Medicina Tropical de Sao Paulo, 38: 29-33.

Rebollar-Téllez, E.A., Reyes, F., Fernández-Salas, I. y Andrade, F.J. (1996c) Abundance and parity rate of Lutzomyia cruciata (Diptera: Psychodidae) in an endemic focus of localized cutaneous leishmaniasis in southern Mexico. Journal of Medical Entomology, 33: 683-685.

Rebollar-Téllez, E.A., Tun-Ku, E., Che, A. y Dzul, F. (2005) Relative abundance of sandfly species (Diptera: Phlebotomidae) in two villages in same area of Campeche in Southern México. Annals of Tropical Medicine and Parasitology, 99: 193-201.

Roberts, D.R. y His, B.P. (1979) An index of species abundance for use with mosquito surveillance data. Environmental Entomology, 8: 1007-1013.

Rodríguez-Rojas, J.J. y Rebollar-Téllez, E.A. (2017) Effect of trapping methods on the estimation of alpha diversity of a phlebotomine sandfly assemblage in southern México. Medical and Veterinary Entomology, 31: 392-401.

Rodríguez-Rojas, J.J., Rodríguez-Moreno, A., Berzunza, M., Gutiérrez-Granados, G., Becker, I., Sánchez-Cordero, V., Stephens, C.R., Fernández-Salas, I. y RebollarTéllez, E.A. (2017) Ecology of phlebotomine sandflies and putative reservoirs host of leishmaniasis in a border area in Northeastern Mexico: implications for the risk of transmission of Leishmania mexicana in Mexico and the USA. Parasite, 24: 33. 
Rojas, E., Scorza, J.V., Morales, G., Morales, C., Barazarte, R. y Torres, A. (2004) Diversity and species composition of sand flies (Diptera: Psychodidae) in a Venezuelan urban focus of cutaneous leishmaniasis. Journal of American Mosquito Control Association, 20: 189-194.

Salomón, O.D., Rossy, G.C., Cousiño, B., Spinelli, GR, Rojas de Arias, A., López del Puerto, D.G. y Ortiz, J.A. (2003) Phlebotominae sand flies in Paraguay. Abundance, distribution in the southeastern region. Memorias do Instituto Oswaldo Cruz, 98: 185-190.

Salomón, O.D., Wilson, M.L., Munstermann, L.E. y Travi, B. (2004) Spatial and temporal patterns of phlebotomine sand flies (Diptera: Psychodidae) in a cutaneous leishmaniasis focus in northern Argentina. Journal of Medical Entomology, 41: 33-39.

Sanchez-García, L., Berzunza, M., Becker, I. y Rebollar-Téllez, E.A. (2010) sand flies naturally infected by Leishmania (L.) mexicana in the peri-urban area of Chetumal city, Quintana Roo, México. Transactions of the Royal Society of Tropical Medicine and Hygiene, 104: 406-411.

Seidelin, H. (1912) Leishmaniasis and babesiasis in Yucatán. Annals of Tropical Medicine and Parasitology, 6: 295-299.

Shannon, C.E. (1948) A mathematical theory of communication. Bell System Technical Journal, 27: 379-423.

Shannon, R. (1939) Methods for collecting and feeding mosquitoes in jungle yellow fever studies. American Journal of Tropical Medicine and Hygiene, 30: 131-138.

Shattuck, G.C. (1938) Chiclero ulcer and other forms of leishmaniasis in America. Carnegie Institute of Washington Publications, 499: 113-140.

Simpson, E.H. (1949) Measurement of diversity. Nature, 163: 688.

Stone, L. y Roberts, A. (1990) The checkerboard score and species distributions. Oecologia, 85: 74-79.

Sudia, W. y Chamberlain, R. (1962) Battery light trap an improved model. Mosquito News, 22: 123-129.

Valderrama, A., Herrera, M. y Salazar, A. (2008) Relación entre la composición de especies del género de Lutzomyia França (Diptera: Psychodidae, Phlebotominae) y los diferentes tipos de bosques en Panamá. Acta Zoológica Mexicana, 24: 67-78.

Van Wynsberghe, N.R., Canto-Lara, S.B., Damían, A., Itzá, M. y Andrade-Narváez, F. (2000) Retention of Leishmania (Leishmania) mexicana in naturally infected rodents from the state of Campeche, México. Memorias do Instituto Oswaldo Cruz, 95: 595-600.

Young, D. (1979) A review of the blood sucking Psychodidae flies of Colombia (Diptera: Phlebotominae and Sycoracinae). IDAS. University Florida, Gainesville. 226 pp.

Young, D.G. y Duncan, M.A. (1994) Guide to the identification and geographic distribution of Lutzomyia sand flies in México, the West Indies, Central and South American (Diptera: Psychodidae). Memoirs of the American Entomological Institute. Gainesville: Associated Publishers. 881 pp. 
